\title{
Post COVID-19 longitudinally extensive transverse myelitis (LETM)-a case report
}

\author{
Seyed Mohammad Baghbanian ${ }^{1,2}\left(\right.$ Farzaneh Namazi $^{1,2}$
}

Received: 6 June 2020 / Accepted: 7 September 2020 / Published online: 18 September 2020

(c) Belgian Neurological Society 2020

\section{Dear editor}

A 53-year-old female was admitted to the neurology ward of Booalisina hospital Sari, Iran after suffering from paraparesis for 2 days. The patient's symptoms started with distal left limb involvement, and after a few hours, the right lower limb was also involved. The symptoms were fully established within 2 days. Three days before the weakness of the lower limbs, the patient suffered from radicular low back pain and transient urinary incontinence. The patient had a history of diabetes mellitus, hypertension, and ischemic heart disease. The systemic examination was normal. Her neurological examination demonstrated asymmetrical hypotonic paraparesis $3 / 5$ power in the proximal and distal part of the right lower limbs and 0/5 power in the proximal and distal part of left lower limbs with areflexia and bilateral Babinski sign. Sensory examination revealed a sensory level at T11-T12 to pinprick testing and bilateral position test impairment in the bilateral lower extremities. The tendon reflexes in both upper limbs and cranial nerve examination were normal. 2 weeks before, the patient had been admitted and treated for COVID-19 and at that time the results of coronavirus RNA nasopharyngeal swab reported positive. Chest CT revealed a patchy ground-glass consolidation in the middle and lower lobes of the right lung (Fig. 1). CSF testing showed 13 cells predominantly lymphocytes with normal protein and glucose. CSF OCB reported negative and the $\operatorname{IgG}$ index was in the upper limit of normal (0.71).

Seyed Mohammad Baghbanian sm.baghbanian@mazums.ac.ir; mohammadbaghbanian@gmail.com

Farzaneh Namazi

farzanehnamazi1363@yahoo.com

1 Department of Neurology, Booalisina Hospital, Faculty of Medicine, Mazandaran University of Medical Sciences, Sari, Iran

2 Neurology Department, Booalisina Hospital, Pasdaran Boulevard, Sari, Iran
Brain MRI was normal and spinal cord MRI showed a longitudinally extensive transverse myelitis in the T8-T10 cord segments (Fig. 2). Without corticosteroid therapy, plasmapheresis was done and paresis recovered to a certain degree. The patient was discharged and referred for rehabilitation treatment.

Central nervous system involvement in COVID disease occurs hematogenously or by neuronal retrograde or antegrade polarization via peripheral nerves, nucleus solitaries of the brain stem, spinal cord, olfactory and trigeminal system [1].

Post-infection myelitis was reported in a confirmed COVID patient with high fever, bone marrow suppression, and liver function failure. It is interpreted that immune damage in the early stage of COVID might be explained the myelitis [2].

In our patient after 14 days of the initial phase of COVID infection, myelitis presented without any signs of acute infection. No significant abnormality was seen in laboratory tests. Liver function tests, Anti NMO Ab, Anti MOG $\mathrm{Ab}$, and CSF PCR for cytomegalovirus and herpes simplex virus were negative. CSF PCR for the COVID-19 virus was negative. Acute disseminated encephalomyelitis (ADEM) which presents after a systemic infection may present with LETM but ADEM without brain involvement is unusual.

IL-6 plays a key role in the second attack of COVID disease which begins 7-14 days after the first respiratory presentation. Although increase IL-6 induces B-cells differentiation and has an anti-inflammatory effect, it could cause acute phase cytokine overproduction and cytokine release syndrome (cytokine storm) associated with fever and multiple organ failure [3]. Guillain-Barré Syndrome associated with COVID infection was defined [4]. An autoimmune antibody reacting to myelin glycoproteins of peripheral nerves has been proposed as a mechanism of Guillain-Barré Syndrome(GBS) [5]. GBS associated with COVID infection raises consideration of COVID triggering possible autoimmunity and similarly induced autoimmunity which might 


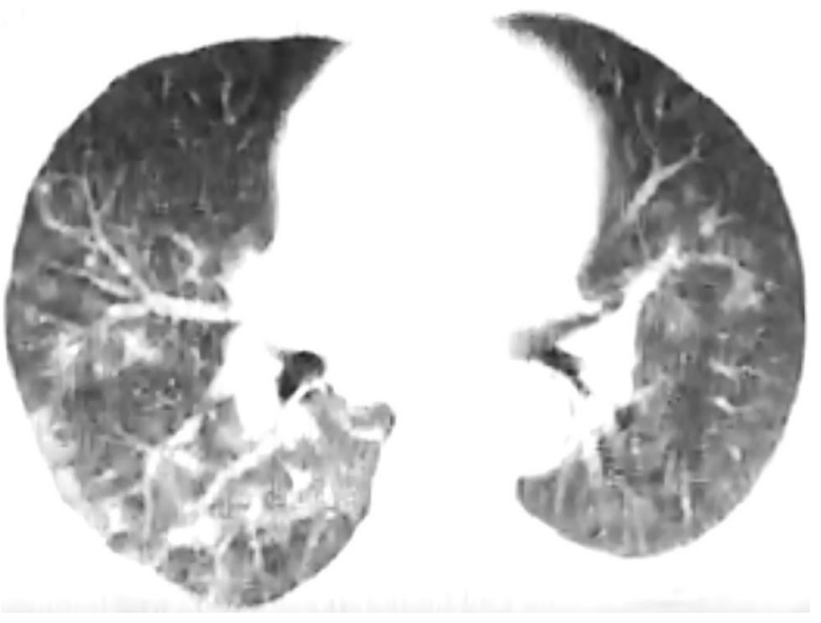

Fig. 1 Middle and lower lob of the lung involvement in high resolution lung CT scan

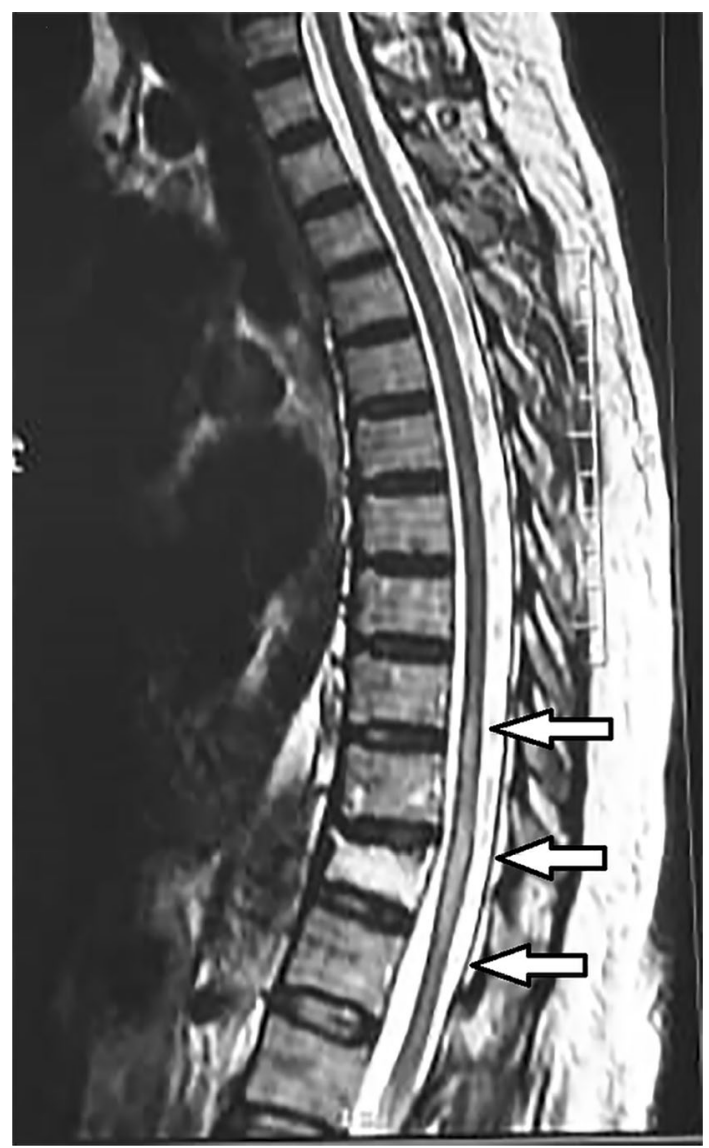

Fig. 2 longitudinally thorasic extensive transverse myelitis T2sagital spinal cord MRI

be the pathogenesis of myelitis associated with COVID. To the best of our knowledge, here in the present study, we have reported the first para infection post-COVID; longitudinally extensive transverse myelitis(LETM). It seems post-COVID CNS autoimmunity is a possible concern after the initial presentation of COVID.

Funding The authors have not declared a specific grant for this research from any funding agency in the public, commercial, or not-for-profit sectors.

\section{Compliance with ethical standards}

Conflict of interest The authors declare that they do not have any conflict of interest.

Ethical approval The Regional Ethics and Hospital Management Committee of Mazandaran. University School of Medicine approved the study (IR.MAZUMS.REC.1399.305).

Informed consent Obtained.

\section{References}

1. Conde Cardona G, Quintana Pájaro LD, Quintero Marzola ID, et al (2020) Neurotropism of SARS-CoV 2: Mechanisms and manifestations. Journal of the Neurological Sciences 412

2. Zhao K, Huang J, Dai D et al (2020) Acute myelitis after SARS-CoV-2 infection: a case report. medRxiv. https://doi. org/10.1101/2020.03.16.20035105

3. Chen C, Zhang XR, Ju ZY, He WF (2020) Advances in the research of cytokine storm mechanism induced by Corona Virus Disease 2019 and the corresponding immunotherapies. Zhonghua shao shang za zhi $=$ Zhonghua shaoshang zazhi $=$ Chin $\mathrm{J}$ Burns 36:E005. https://doi.org/10.3760/cma.j.cn501120-20200 224-00088

4. Toscano G, Palmerini F, Ravaglia S et al (2020) Guillain-Barré syndrome associated with SARS-CoV-2. N Engl J Med. https:// doi.org/10.1056/nejmc2009191

5. Yuki N, Hartung HP (2012) Guillain-Barré syndrome. N Engl J Med 366:2294-2304

Publisher's Note Springer Nature remains neutral with regard to jurisdictional claims in published maps and institutional affiliations. 